\title{
L'enseignement en mode synchrone des activités physiques et sportives dans un contexte de confinement
}

\section{J. Alem \& H. Hariti}

DRHJ/RDRS 2021, 4, pp.185-194

Jaouad Alem, Ph.D.

Professeur agrégé, jalem@laurentian.ca

Groupe de Recherche sur l'Évaluation et le Développement des Compétences en Activité Physique et en Santé (GRÉDCAPS), École de kinésiologie et des sciences de la santé.

Faculté de la santé, Université Laurentienne, Sudbury (ON), Canada.

Hakim Hariti, Ph.D.

Professeur titulaire, hariti.hakim@univ-alger3.dz

Laboratoire Sciences et Pratiques des Activités Physiques Sportives et Artistiques SPAPSA Institut de l'Éducation Physique et Sportive (IEPS), Université d'Alger 3 (Alger), Algérie.

\section{Résumé}

Dans un contexte de confinement, jusqu'à quel point est-il possible de développer en mode synchrone des apprentissages physiques, des apprentissages moteurs ou des apprentissages affectifs ? Une question fermée, dont les réponses étaient encodées sur une échelle de type Likert en quatre points, a permis de mesurer la perception des acteurs sociaux engagés dans le développement physique, moteur et affectif des participants. L'échantillon était composé de 102 acteurs sociaux sportifs originaires du Maroc et de l'Algérie et âgés de 9 à 64 ans. Les acteurs étaient des formateurs, des administrateurs, des sportifs ou des parents. La question a fait l'objet d'une analyse de variance selon trois variables nominales (genre, statut, pays) et d'une corrélation de Pearson entre l'âge et les trois types de développement souhaités. Quelques hypothèses d'action sont envisagées pour faire face aux défis didactiques que pose ce type particulier d'enseignement en situation de confinement.

Mots clés : enseignement en mode synchrone, apprentissages physiques, moteurs, affectifs.

\section{Abstract}

In a context of isolation, to what extent is it possible to develop synchronous physical learning, motor learning or affective learning? Using a closed question, the responses of which were encoded on a four-point Likert-type scale, the researchers measured the perception of people involved in the physical, motor and emotional development of the participants. The sample was composed of 102 people involved in sports from Morocco and Algeria, aged 9 to 64. They were trainers, administrators, athletes or parents. The question was analyzed for variance by three nominal variables (gender, status, country) and a Pearson correlation between age and the three desired types of development. Some hypotheses are considered with respect to the didactic challenges of teaching in isolation.

Keywords: teaching in synchronous mode, physical, motor, emotional learning.

\section{Introduction et problématique}

Dans un contexte de confinement des formateurs et des étudiants, les formations sont assurées en mode synchrone ou en mode asynchrone. Ceci les oblige à repenser leurs façons d'enseigner et d'apprendre, pouvant causer certaines frustrations (Audet, 2012) dans la mesure ou la communication en ligne ne paraît pas remplacer l'intérêt des contacts en face à face (Conrad, 2005). Dans ce contexte, beaucoup d'activités physiques et sportives (APS) 
pourraient disparaitre en formation à distance (FAD). D'ailleurs Huston (1997) puis Courtney, Stanley et Muggy (1999) identifient la motivation comme un des facteurs de succès pour les apprenants dans un environnement de FAD.

\section{Recension de la documentation}

Les difficultés de financement de la formation universitaire ont généré de nouveaux modèles d'apprentissage comme la formation à distance pour répondre à différents besoins éducatifs. Dans ce contexte, la formation à distance est considérée comme essentielle à la stratégie à long terme par une majorité d'établissements (Tichavsky, Hunt, Driscoll et Jicha, 2015).

Bien avant le confinement, certaines recherches estimaient déjà qu'une offre de formation à distance devrait être adaptée aux besoins et aux disponibilités des personnes ne pouvant pas se déplacer sur le campus (Bindé, 2003; Doray, Kamanzi, Laplante et Street, 2012; Head, Van Hoeck et Garson, 2015). D'autres chercheurs comme Downes (2012) et Siemens (2005) soutiennent une approche dite connectiviste du savoir ou l'apprentissage réside dans la capacité de créer des connexions et de partager des informations à travers elles.

Les difficultés de communication et d'interaction causées par la FAD exacerbent l'émergence d'émotions négatives qui deviennent alors un vecteur de frustrations (Ragoonaden et Bordeleau, 2000; Ng, 2001). Do et Schallert (2004) ainsi que Pekrun, Goetz, Titz et Perry (2002), révèlent qu'en situation d'apprentissage à distance, les apprenants rapportent avoir éprouvé une gamme d'émotions, dont la frustration est l'émotion négative qui est la plus fréquemment rapportée. La frustration des apprenants peut être causée par des facteurs internes, comme le sentiment d'auto-efficacité et l'humeur, ou externes, comme les facteurs situationnels liés à l'importance de la tâche et le temps perdu (Bessiere, Newhagen, Robinson et Shneiderman, 2006). Cette frustration peut entraîner une diminution de la motivation intrinsèque et par le fait même une diminution de l'engagement académique des étudiants (Papi, Mendoza, Brassard, Bédard, et Sarpentier, 2017) et peut conduire les étudiants à abandonner leurs études (Conrad, 2002).

Selon la littérature, l'évaluation en ligne par les pairs (Hadi Bouzidi et Jaillet, 2007) ou encore l'enseignement réciproque de Mosston et Ashworth (2006) semblent être des stratégies pédagogiques efficaces pour obtenir, développer et évaluer la motivation des participants. En effet, la qualité de l'évaluation par les pairs est comparable à celle des professeurs à condition que les critères d'évaluation soient bien expliqués (Alem, 1993).

Pour rappel, une prestation synchrone signifie que le cours est offert en temps réel, par exemple, une séance sur Zoom en direct, au cours de laquelle l'enseignant examine la matière et permet les interactions avec les étudiants. Par contre, une prestation asynchrone signifie que la matière du cours est accessible à tout moment, c'est-à-dire que l'enseignant peut, par exemple, publier une séance enregistrée sur D2L et demander aux étudiants de répondre, à même la plateforme, à des questions posées dans le cadre d'un forum de discussion.

Dans la FAD en mode synchrone, l'ensemble du contenu et des activités des cours sont donc mis à disposition des étudiants qui doivent participer à certains enseignement ou échanges à des temporalités et selon des moyens technologiques prévus dans le dispositif (Gérin-Lajoie et Potvin, 2011). Par exemple, lors d'une séance sur Zoom en mode synchrone, les participants peuvent poser des questions et interagir avec leurs pairs. 
Par contre, lors de la FAD en mode asynchrone, l'ensemble du contenu et des activités des cours sont mis à la disposition des étudiants qui peuvent choisir le moment de les réaliser. Les étudiants ont le choix de communiquer avec le formateur ou avec leurs pairs par l'envoi de messages (Gérin-Lajoie \& Potvin, 2011). Le formateur peut, par exemple, publier une séance enregistrée sur une plateforme pédagogique ou demander aux participants de répondre sur la même la plateforme, à des questions posées dans le cadre d'un forum de discussion. Les cours en mode asynchrone permettent aux étudiants qui vivent dans différents fuseaux horaires ou dont l'emploi du temps personnel est décalé, d'accéder aux contenus à l'heure qui leur convient. Dans ce mode d'enseignement, il y a deux particularités susceptibles d'influencer la motivation des étudiants. Premièrement, le langage écrit remplace la communication orale des cours en présentiel. Deuxièmement, la distance temporelle engendre des délais dans les réponses que les étudiants souhaitent obtenir de leurs questions ou encore une communication asynchrone (Cheng-Yuan, 2000).

Selon Fekkak (2020), l'impact du confinement sur la forme physique, l'état mental et la technique des sportifs se traduit par la perte d'endurance et par la baisse de la masse musculaire. En effet, durant le confinement, les sportifs se contentent souvent du vélo stationnaire (1h30) pour effectuer leurs séances de cardio, indispensable pour améliorer les performances physiques et techniques. Ceux qui ne disposent pas de vélo stationnaire sont limités à de simples exercices de saut à la corde, qui à leur tour, aident à améliorer les performances mais restent insuffisants pour des sportifs de haut niveau. Plusieurs ont été dans l'incapacité d'effectuer des exercices physiques explosifs permettant de travailler l'intensité de l'effort qui peut être croissante, décroissante ou encore fluctuante. En effet, les entraînements à domicile ne permettent pas aux sportifs d'atteindre le degré d'intensité souhaité. Ces entraînements à domicile ont eu également un impact négatif sur le niveau technique des athlètes, notamment dans les disciplines qui nécessitent des entrânements collectifs comme le judo et la lutte. Privés d'occasion de compétition, certains athlètes en arts martiaux notamment les karatékas et les taekwondoïstes, perdent leur rapidité et leur vivacité. Ces athlètes perdent, au fil du temps, la vitesse de réaction dans l'attaque ou la défense qu'ils avaient aiguisée auparavant, et trouvent plus de difficultés à assurer de bons appuis sur le tapis. Dans le cas de certains sports comme la natation, dont l'entraînement ne peut pas être effectué en dehors des piscines, le confinement des nageurs a également impacté négativement leur condition physique. Cet effet négatif se manifeste par une perte de rythme en l'absence d'entraînement en groupe et surtout une perte du volume de la masse musculaire.

A l'Université Laurentienne, comme c'est le cas dans beaucoup d'autres universités, la plupart des cours ont été offerts à distance durant la session d'automne 2020 et seront probablement offerts en mode hybride, c'est-à-dire combinant les prestations synchrone et asynchrone pour la session d'hiver 2021. Cependant, L'offre de cours en ligne pose particulièrement problème pour les stages pratiques, les activités physiques et sportives ainsi que pour les laboratoires.

Dans ce contexte particulier, jusqu'à quel point les acteurs sociaux impliqués dans la formation perçoivent-ils qu'il est possible de développer en mode synchrone les apprentissages physiques, moteurs et/ou affectifs souhaités dans l'enseignement des activités 
physiques et sportives ? C'est la question principale de recherche à laquelle souhaite répondre les auteurs.

Selon Parlebas (1987), le sport est l'ensemble fini et dénombrable des situations qui possèdent quatre caractéristiques incluant des situations d'actions motrices qui sont codifiées, font l'objet de compétitions et sont institutionnalisées.

Le paragraphe suivant décrit la méthodologie ayant permis de répondre à la question de recherche.

\section{Méthodologie}

La prochaine section présente les différentes composantes de la méthodologie retenue. Essentiellement, il s'agit du type d'enquête, des participants à l'étude, de la méthode de collecte de données et du mode d'analyse de ces données

\section{Type d'enquête}

\section{Participants}

L'échantillon était composé de 102 acteurs sociaux sportifs, originaires du Maroc $(n=75)$ et de l'Algérie $(n=27)$ dont 38 femmes/filles, et 64 hommes/garçons, âgés entre 9 et 64 ans. Les acteurs étaient soit des formateurs $(n=34)$, des administrateurs $(n=24)$, des sportifs $(n=19)$ ou des parents $(n=25)$. Le tableau suivant présente les caractéristiques démographiques de l'échantillon.

Tableau 1 : Les caractéristiques démographiques de l'échantillon

\begin{tabular}{rr|c|c|c|c|}
\cline { 2 - 6 } & & formateurs & administrateurs & sportifs & Parents \\
\hline Fille/femme & Algérie & 3 & 3 & 4 & 3 \\
& Maroc & 9 & 6 & 4 & 6 \\
Garçon/homme Algérie & 4 & 4 & 3 & 3 \\
Maroc & 18 & 11 & 8 & 13 \\
\hline
\end{tabular}

\section{Méthode de collecte des données}

La question fermée a été administrée au cours du mois d'avril 2020 en format papier, Toutes les mesures visant à assurer la sécurité des participants par rapport à la Covid-19 ont été prisent.

Cette question fermée a été formulée de la manière suivante : quels sont, selon vous, les types de développement qui peuvent assez facilement et sans grand danger être enseignés en ligne et en mode synchrone ?

La question fermée dont les réponses étaient encodées sur une échelle de Likert en quatre points $(1=$ très difficilement, $4=$ très facilement $)$ mesurait la perception des acteurs sociaux engagés dans le développement physique, moteur et affectif des participants.

\section{Mode d'analyse des données}

La question a fait l'objet d'une analyse de variance (ANOVA) selon trois variables nominales (genre, statut, pays) et d'une corrélation de Pearson entre l'âge des acteurs sociaux 
Diversity of Research in Health Journal / Revue de la Diversité de la Recherche en Santé Vol 4, January/ Janvier 2021 - ISSN: 2561 -1666 DOI : 10.28984/drhj.v4i1.322

et les trois types enseignés en APS en ligne et en mode synchrone, à savoir : (1) le développement moteur (les techniques), (2) le développement physique (vitesse, adresse, résistance et force) et (3) le développement affectif (socialisation, développement de bonnes attitudes, etc.).

Pour comparer les moyennes des groupes, nous avons utilisé des tests T de Student pour deux échantillons indépendants. Nous avons testé l'homogénéité des variances des groupes avec le test de Levene (1960). Quand celui-ci ne rejetait pas l'hypothèse nulle de l'égalité des variances, nous avons utilisé le test alternatif $\mathrm{F}^{*}$ de Morton Brown et Alan Forsythe (2012) pour comparer les moyennes. Ce test considère la médiane au lieu de la moyenne pour calculer les scores déviations : il est considéré plus robuste à la non-normalité des scores selon Conover, Johnson et Johnson (1981) et Olejnik et Algina (1987).

Le logiciel statistique utilisé était SPSS, version 22.

\section{Résultats et analyse}

Comme le montre le graphique 1, il apparait que les développements physiques et affectifs sont perçus significativement plus difficile à évaluer par les acteurs sociaux marocains que par leurs homologues algériens (respectivement 2,68 contre 3,52 et 3,18 contre $3,78)$.

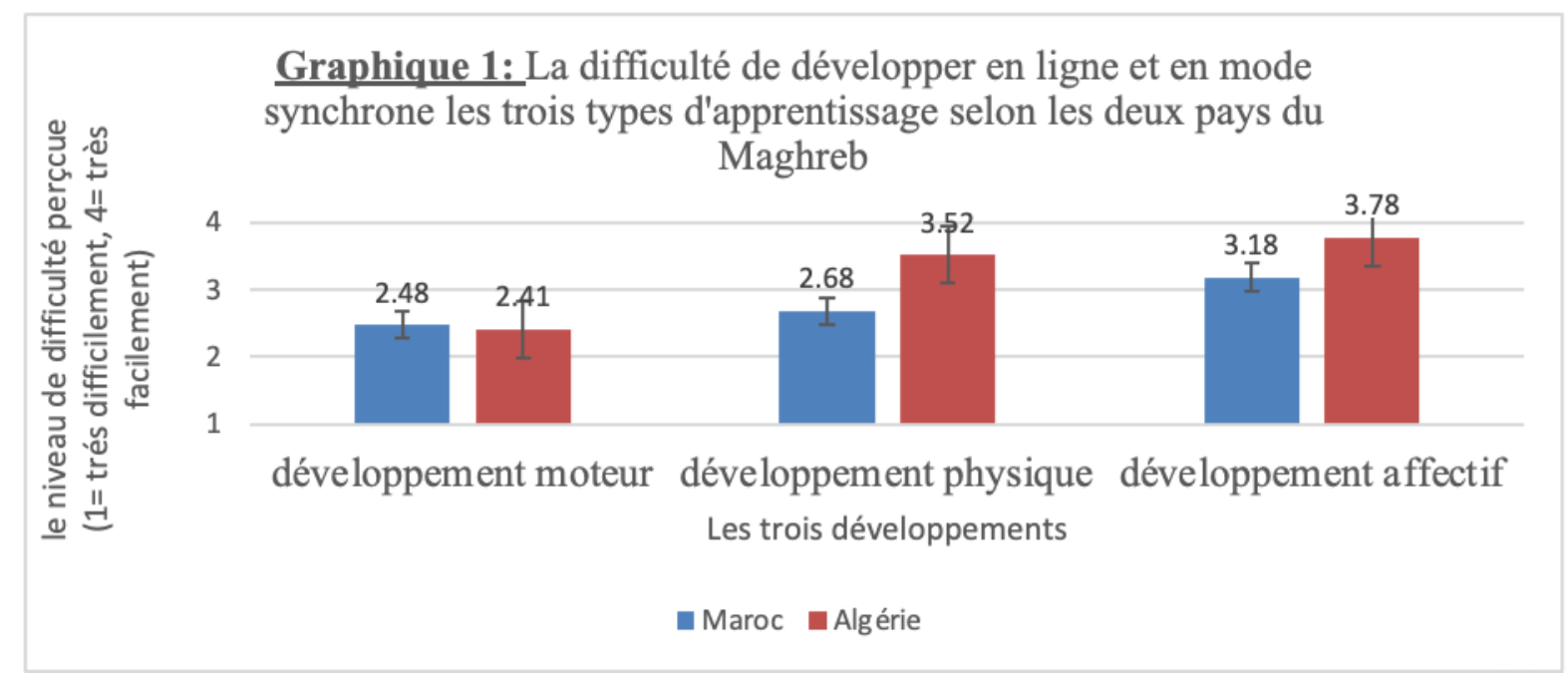

Le graphique 2 montre que l'effet du genre n'est significatif que pour l'évaluation du développement affectif en faveur du genre féminin (3,63 contre 3,21) qui semble plus prédisposé à bénéficier de ce type de formation pour ce type de développement. 
Diversity of Research in Health Journal / Revue de la Diversité de la Recherche en Santé Vol 4, January/ Janvier 2021 - ISSN: 2561 -1666 DOI : 10.28984/drhj.v4i1.322

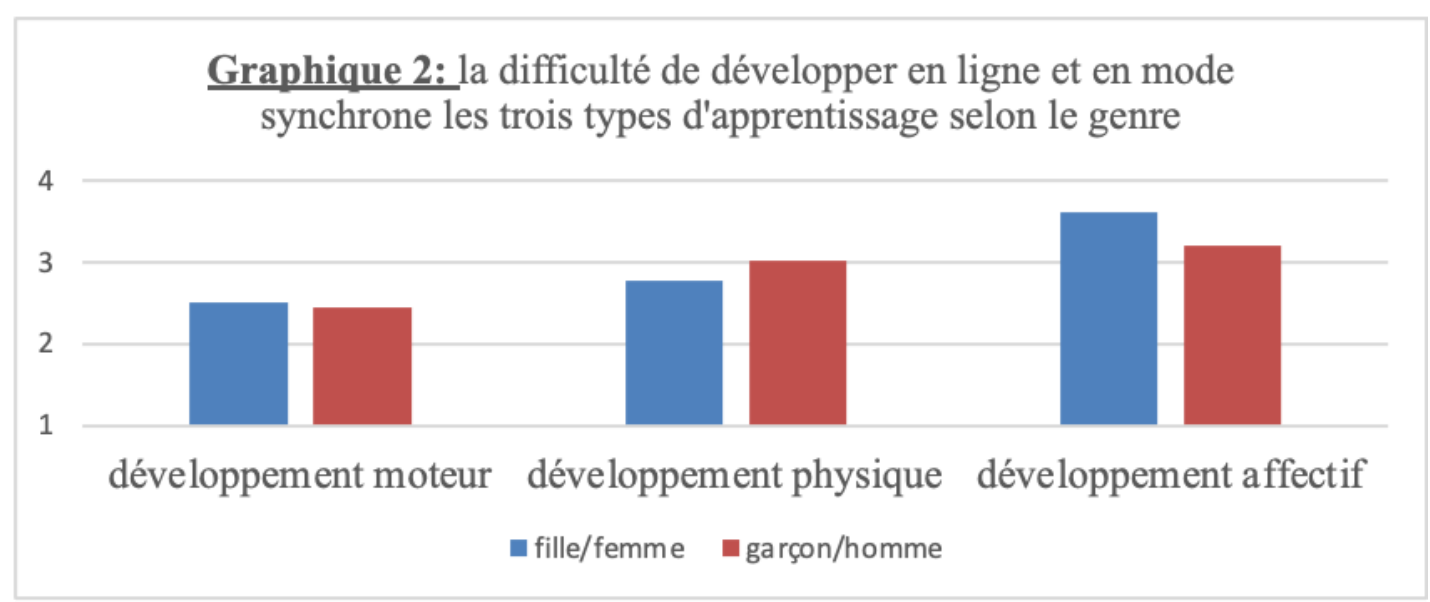

Par contre, comme le montre le graphique 3, l'effet du statut de l'acteur social n'est significatif ni pour le développement physique $(\mathrm{p}=0,17)$, ni pour le développement moteur ( $\mathrm{p}$ $=0,24)$ ni pour le développement affectif $(\mathrm{p}=0,10)$.

Graphique 3 : les effets du statut social sur la perception de la difficulté à développer les trois types d'apprentissage

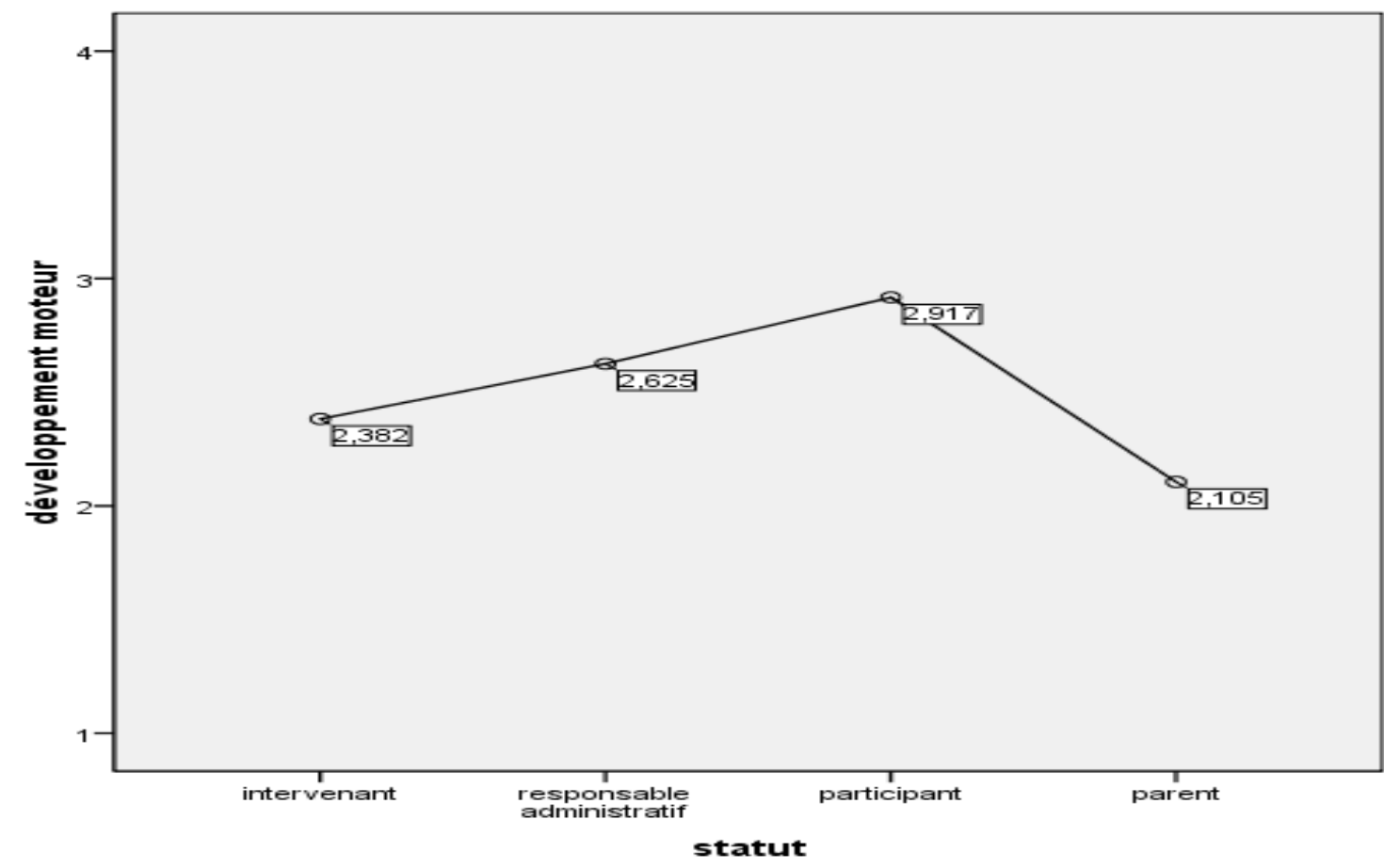


Diversity of Research in Health Journal / Revue de la Diversité de la Recherche en Santé

Vol 4, January/ Janvier 2021 - ISSN: 2561 -1666 DOI : 10.28984/drhj.v4i1.322

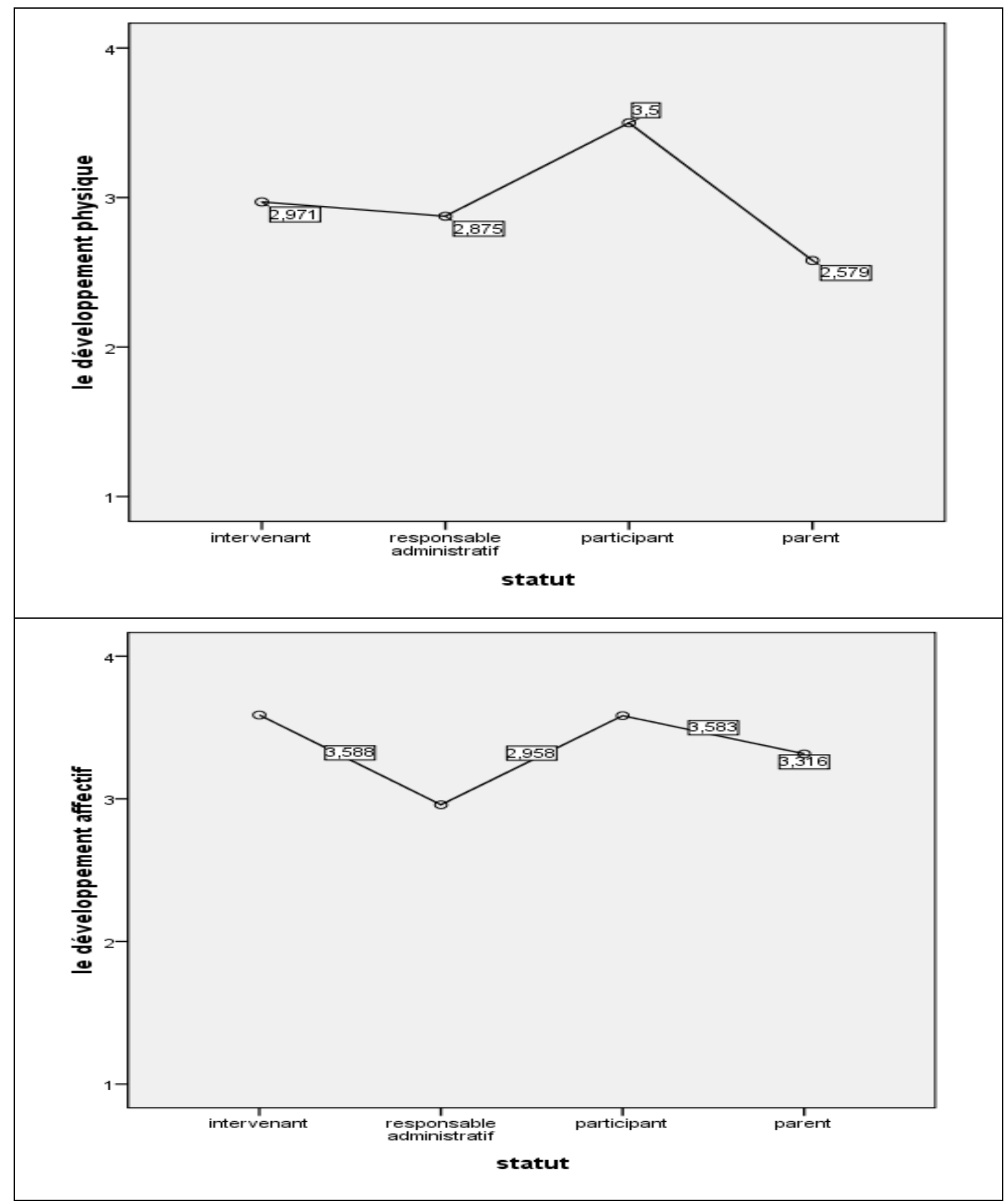

Enfin, les 'jeunes' acteurs sociaux perçoivent moins difficile de participer à des formations en mode synchrone pour des développements physiques, mais la corrélation est plutôt faible $\left(\mathrm{R}=-0,22^{*} ; \mathrm{N}=88\right.$ ) comme le montre le tableau comparatif 2 . 
Tableau 2: la perception de la difficulté de développer les trois types

\begin{tabular}{|l|c|c|c|}
\hline \multicolumn{4}{|c|}{ d'apprentissages selon l'âge } \\
\hline & $\begin{array}{c}\text { Développement } \\
\text { moteur }\end{array}$ & $\begin{array}{c}\text { Développement } \\
\text { physique }\end{array}$ & $\begin{array}{c}\text { Développement } \\
\text { affectif }\end{array}$ \\
\hline Age / Corrélation de Pearson &,- 10 &,$- 22^{*}$ &,- 13 \\
Sig. (2-tailed) &, 38 &, 04 &, 22 \\
N & 88 & 88 & 88 \\
\hline
\end{tabular}

\section{Conclusion et recommandations}

En situation de confinement dû à la pandémie de la covid19, les solutions envisagées par les universités sont de reporter à une date ultérieure l'enseignement en présentiel des APS et à offrir l'enseignement en mode asynchrone seulement, soit d'offrir en mode synchrone des APS dites « douces » telles que le yoga, la dance en ligne ou la gymnastique au sol adaptée.

Enfin, il semble difficile de renforcer la motivation et la qualité de la participation sans noter les étudiants de façon formelle et sommative.

Il conviendrait de tester à nouveau les mêmes hypothèses de recherche mais en tenant compte d'autres variables potentiellement déterminantes des trois développements souhaités telles que la culture professionnelle des intervenants, leur genre et leur degré d'expérience professionnelle.

\section{Références}

Alem, J. (1993). L'influence l'évaluation par les pairs sur l'apprentissage du lancer du poids par des élèves du secondaire. Mémoire de maîtrise. Département des sciences de l'activité physique. Université Laval. Québec Bibliothèque nationale du Canada.

Audet, L. (2012). Profil, bilan et perspectives de l'apprentissage à distance. Réseau d'enseignement francophone à distance du Canada.

http://archives.refad.ca/pdf/LAudet_Memoire_PPB_v20120227.pdf

Bessiere, K., Newhagen, J. E., Robinson, J. P., et Shneiderman, B. (2006). A model for computer frustration: The role of instrumental and dispositional factors on incident, session, and post-session frustration and mood. Computers in Human Behavior, 22(6), 941-961.

Bouzidi, L.H, et Jaillet, A. (2007). L'évaluation par les pairs pourra-t-elle faire de l'examen une vraie activité pédagogique ? Communication présentée au congrès de l'EIAH, Lausanne, Suisse, 27-28-29 juin.

Bindé, J. (2003). Mondialisation et contrat culturel : vers l'éducation pour tous tout au long de la vie ? . In J. C. Ruano-Borbalan,. (dir.), Transmettre en éducation, formation et organisation (p. 63-98). Paris: Demos.

Brown, M., et Forsythe, A. (2012). The small sample behavior of some statistics with test of equality of several means. Tecnometrics, 16, (1), 129-132.

Cheng-Yuan, C.L. (2000). Student motivation in the online learning environment. Journal of Educational Media and Library Sciences. 37(4), 367-375. 
Diversity of Research in Health Journal / Revue de la Diversité de la Recherche en Santé

Vol 4, January/ Janvier 2021 - ISSN: 2561 -1666 DOI : 10.28984/drhj.v4i1.322

Conover W., Johnson M., et Johnson, M. (1981). A comparative study of test for homogeneity if variance with applications to the outer continental shelf bidding data. Tecnometrics, 23, (4), 357-361.

Conrad, D. L. (2002). Engagement, excitement, anxiety, and fear: Learners' experiences of starting an online course. American Journal of Distance Education, 16(4), 205-226.

Conrad, D.L. (2005). Building and Maintaining Community in Cohort-Based Online Learning. Journal of Distance Education, 20(1), 1-20.

Courtney, S. V., Stanley, L.J., et Muggy, V. (1999). Characteristics of Adults as Learners and Implications for Computer-Based Systems for Information and Instruction. U.S.: Nebraska.

Do, S., et Schallert, D. (2004). Emotions and classroom talk: Toward a model of the role of affect in students' experiences of classroom discussions. Journal of Educational Psychology, 96(4), 619-634.

Doray, P., Kamanzi, P.C., Laplante, B. et Street, M.C. (2012). Les retours aux études postsecondaires : une expression de l'éducation tout au long de la vie ? Formation emploi, (120). 75-100.

Downes, S. (2012). Connectivism and Connective Knowledge: essays on meaning and learning networks. National Research Council Canada. https://www.oerknowledgecloud.org/archive/Connective_Knowledge-19May2012.pdf

Fekkak, H. (2020). Impact du confinement sur le physique des sportifs : Quatre questions au directeur Hassan Hnique Fekkak. http://www.mapexpress.ma/actualite/sports/impact-du-confinement-physique-sportifsquatre-questions-au-directeur-technique-hassan-fekkak/

Gérin-Lajoie, S., et Potvin, C. (2011). Évolution de la formation à distance dans une université bimodale. Distances et Savoir, 9(3), 349-374.

Head, A., Van Hoeck, M., et Garson, D. (2015). Lifelong learning in the digital age: A content analysis of recent research on participation. First Monday, 20 (2). https://doi.org/10.5210/fm.v20i2.5857

Huston, J. L. (1997). Factors of success for adult learners in an interactive compressed video distance learning environment. US: U Kentucky.

Levene, H. (1960). Robust tests for equality of variances. In I. Olkin, H. Hotelling et al. (eds): Contributions to Probability and Statistics: Essays in Honor of Harold Hotelling (pp. 278-292). Palo Alto, California: Stanford University Press.

Mosston, M., et Ashworth, S. (2006). Le Spectre des styles d'enseignement : application à l'enseignement de l'éducation physique (5e éd.). North Hatley, Canada : Éditions C. \& C.

Ng, K.C. (2001). Using e-mail to foster collaboration in distance education. Open Learning, 16(2), 191-200.

Olejnik, S. et Algina, J. (1987), «Type I Error Rates and Power Estimates of Selected Parametric and Nonparametric Tests of Scale », Journal of Educational Statistics, 12 (1), 45-61.

Papi, C., Mendoza, G. A., Brassard, C., Bédard, J.L., et Sarpentier, C. (2017). L’interaction en formation à distance: entre théories et pratiques. TransFormations-Recherches en éducation et formation des adultes, (17). 
Diversity of Research in Health Journal / Revue de la Diversité de la Recherche en Santé

Vol 4, January/ Janvier 2021 - ISSN: 2561 -1666 DOI : 10.28984/drhj.v4i1.322

Parlebas, P. (1987). Éléments de sociologie du sport. [compte-rendu]. Dir. Alain Degenne. Revue française de sociologie (pp. 547-550). ESF Éditeur.

Ragoonaden, K., et Bordeleau, P. (2000). Collaborative learning via the Internet. Educational Technology \& Society, 3(3), 361-369.

Siemens, G. (2005). Connectivism: A learning theory for the digital age. International Journal of Instructional Technology and Distance Learning, 2(1), 3-10.

Tichavsky, L. P., Hunt, A. N., Driscoll, A., et Jicha, K. (2015). It's Just Nice Having a Real Teacher": Student Perceptions of Online versus Face-to-Face Instruction. International Journal for the Scholarship of Teaching and Learning, 9(2). https://doi.org/10.20429/ijsotl.2015.090202 